\title{
Depressão em idosos institucionalizados: revisão sistemátical
}

\author{
Resumo
}

Wanaline Fonsêca*; Camila Franco**

A institucionalização para idoso é uma opção muito procurada quando os familiares não aceitam a função de cuidadores. Ao ser institucionalizado o idoso é obrigado a adaptar-se as rotinas de horários, a divisão do ambiente com pessoas desconhecidas, distância da família dentre outros, o que pode acarretar em problemas psicológicos, como a depressão. O estudo teve como objetivo identificar se idosos institucionalizados apresentam depressão. Para construção do estudo foi realizado uma pesquisa bibliográfica, abrangendo artigos científicos entre os anos de 2009 a 2017. Na maioria dos estudos analisados, foi observado um grande número de depressão em idosos institucionalizados devido a fatores como ausência de suporte familiar, limitação e/ou dependência, insatisfação com a instituição e a solidão. Entre os principais sintomas da depressão evidenciados foram a falta de apetite, fadiga acentuada, baixa autoestima, insegurança, diminuição da capacidade de concentração, isolamento social, dentre outros. Dentre os idosos institucionalizados com depressão, a maioria são do gênero feminino, não recebiam visitas familiares, não saíam da instituição, não faziam exames de rotina, e exerceram no passado ocupações de menor complexidade. Desta forma faz-se necessário que os profissionais envolvidos no cuidado ao idoso institucionalizado tenham habilidades e conhecimento para reconhecer e intervir apropriadamente nos casos em que o indivíduo está em sofrimento psíquico.

Palavras-chave: Depressão. Idosos. Institucionalização. Enfermagem.

\section{Introdução}

Envelhecer não se trata de uma fatalidade biológica, e/ou de uma ação psicológica, mas se trata de um conjunto de atitudes, probabilidades, crendice e conceitos da sociedade das mais diversas culturas onde o idoso está inserido (HARTMANN JUNIOR; GOMES, 2014). É cultural a sociedade associar o idoso a dependência e a doença, em consequência disso, o indivíduo senil na maioria das vezes é condenado à solidão ou ao estigma de ser "algo" problemático acrescido ainda das dificuldades financeiras presentes na grande maioria desta população (NETO; CORTE-REAL, 2011).

Dados do Ministério da Saúde mostram que cerca de 50 a $60 \%$ dos casos de

* Enfermeira. Especialização em Enfermagem Cirúrgica pelas Faculdades Integradas de Jacarepaguá, Rio de Janeiro/RS, Saúde e Qualidade de Vida e Enfermagem do Trabalho pela Faculdade Futura, Votuporanga/ SP, e Enfermagem em Pediatria e Neonatologia pela Faculdade Unyleya, Caxias do Sul/RS. Mestranda do Programa de Pós-Graduação de Alimentos, Nutrição e Saúde pela Universidade Federal da Grande Dourados, Dourados/MS.. E-mail: wanalinefonseca@hotmail.com.

** Enfermeira. Cursa especialização em Enfermagem em Pediatria e Neonatologia pela Faculdade Venda Nova do Imigrante, Venda Nova do Imigrante/ES. E-mail: mylafran@gmail.com.

$\rightarrow$ http://dx.doi.org/10.5335/rbceh.v16i3.9081 
depressão não são detectados, em parte por algumas pessoas idosas julgarem ser inútil relatar a dificuldade para $o$ profissional de saúde, concomitante a isso, se estes profissionais não estiverem vigilantes a identificação de sinais e sintomas, certamente o diagnóstico passará despercebido. Os fatores que desencadeiam esse comportamento são a falta de treinamento, tempo, descrença em relação a efetividade do tratamento, dentre outros (BRASIL, 2007).

A depressão é atualmente um problema que atinge todas as faixas etárias, no entanto, em idosos há uma prevalência maior tendo em vista as mudanças fisiológicas associadas as perdas sentimentais ao longo da vida, porém o diagnóstico costuma assumir formas atípicas sendo assim, a descoberta e consequente tratamento torna-se difícil (MARTINS, 2008).

A depressão tem sido caracterizada como uma síndrome que envolve inúmeros aspectos etiopatogênicos clínicos e de tratamento, sobretudo na população idosa. Associando-se a patologias clínicas gerais e a anormalidades estruturais e funcionais do cérebro, tais como Parkinson, Alzheimer, corpos de Lewy, sequelas de AVC, dentre outras e caso não seja tratada aumenta o risco de morbidade e de mortalidade, principalmente em idosos hospitalizados com enfermidades gerais. Muitos são os componentes que configuram as causas de depressão no idoso, podendo ser associados a fatores genéticos e ambientais, como luto e abandono, incapacitação funcional e geralmente a doença surge em um contexto de perda da qualidade de vida associada ao isolamento social e ao surgimento de doenças clínicas graves (STELLA et al., 2002).

O presente estudo teve por objetivo identificar se o idoso institucionalizado apresenta depressão. Diante da problemática, faz se necessário a realização de estudos como esse, com o objetivo de verificar se há a associação entre o surgimento da depressão no idoso e o processo de institucionalização. Esta pesquisa trará grandes benefícios para a comunidade acadêmica e profissional da saúde, tendo em vista que através do seu resultado será possível verificar as principais causas da depressão em idosos institucionalizados, os fatores contribuintes para o agravo da depressão, estabelecer a relação entre depressão e o afastamento do convívio social, melhorando assim, a qualidade e foco do atendimento de cada profissional e a qualidade de vida dos idosos institucionalizados.

\section{Introdução}

Idoso

A Organização Mundial da Saúde (OMS) define o idoso a partir da idade cronológica, com 60 anos ou mais, em países em desenvolvimento e com 65 anos ou mais em países desenvolvidos. É relevante reconhecer que a idade cronológica não é um marcador obrigatório para as mudanças que conduzem o envelhecimento. Existem diferenças significativas que estão relacionadas ao 
estado de saúde, participação e níveis de independência entre pessoas que possuem a mesma idade. A distinção entre idosos jovens, idosos velhos e idosos mais velhos auxilia no entendimento de que o envelhecimento pode ser consequência das experiências passadas, da forma como se vive e se administra a vida no presente e de expectativas futuras; é, portanto, uma integração entre as vivências pessoais e o contexto social e cultural, envolvendo aspectos biológicos, cronológicos, psicológicos e sociais (BRASIL, 2005).

Segundo dados do Instituto Brasileiro de Geografia e Estatística (IBGE) no Brasil, encontram-se aproximadamente 30 milhões de pessoas com idade igual ou superior a 65 anos (BRASIL, 2017). Nos últimos 60 anos o número de pessoas com mais de 60 anos apresentou um crescimento de nove vezes. Em 1940 era de 1,7 milhões e em 2000, de 14,5 milhões. Para 2020 é projetado um contingente de aproximadamente 30,9 milhões de pessoas com mais de 60 anos (BELTRÃO, CAMARANO; KANSO, 2004).

Envelhecer é um processo multifatorial e subjetivo, isto é, cada indivíduo tem seu modo particular de envelhecer. Desta forma, é importante considerar às condições biológicas ligadas ao avanço da idade, acarretando no declínio do funcionamento das funções orgânicas, fatores ligados ao meio externo, que contribuem para a preservação vitalícia do idoso e, o contexto social (DIAS, 2007).

Existem dois conceitos que definem o envelhecimento: a senescência e a senilidade. A senescência é caracterizada pelo período em que transcorre gradualmente um deterioramento físico e mental, trata-se de um processo natural do ciclo da vida. Já a senilidade refere-se à fase em que o declínio físico é mais acentuado e acompanhado de desorganização mental, o qual enquadra-se num processo patológico (JACOB FILHO, 1998). O idoso poderá apresentar falhas em seu desempenho cognitivo e físico como problemas de memória e concentração, necessitando de orientação e atenção. E, na parte funcional há perda da independência e autonomia, necessitando de auxílio para desempenhar suas atividades básicas do cotidiano (DIAS, 2007).

A institucionalização é um processo através do qual são criadas regras no pensamento e na ação social, que consideram o sistema de crenças e estruturas culturais. Pode-se dizer que o processo de institucionalização é o mecanismo de seleção e retenção evolutiva, uma forma de obter características organizacionais que levam à criação de uma instituição (TRAVASSOS, 2012).

As Instituições de Longa Permanência para Idosos (ILPIS) são instituições governamentais ou não governamentais, destinadas a serem domicílios coletivos de pessoas com idade igual ou superior a 60 anos, com ou sem suporte familiar, em condição de liberdade, dignidade e cidadania (BRASIL, 2005). De acordo com o regulamento técnico da $\mathrm{RDC}$ $283 / 2005$, as ILPIS podem ser criadas somente após seguir padrões e promover aos seus residentes exercício dos direitos humanos, garantir a identidade e a privacidade da pessoa idosa, assegurando 
um ambiente de respeito e dignidade, promover a integração dos residentes nas atividades desenvolvidas, garantir e incentivar as relações intergeracionais, desenvolver ações que estimulem a autonomia, oferecer programas de cultura e lazer e proporcionar palestras e eventos para combate à violência e violação de direitos civis contra a pessoa idosa (BRASIL, 2005; FRIAS, 2015).

As ILPIS também devem ser legalmente constituída, através de documentos como estatuto registrado, registro de entidade social e regimento Interno, além de constituir uma equipe que desempenhem tarefas fundamentais, entre eles, um responsável técnico para a instituição e cuidadores que variam em quantidade segundo os requisitos impostos na RDC 283/2005 (BRASIL, 2005; FRIAS, 2015).

Em tempos passados, as ILPIS eram definidas como asilos, atualmente são definidas como instituições de saúde, devido a necessidade do trabalho de profissionais da saúde para tratar das modificações resultantes da geriatria ou alterações psicológicas (CREUTZBERG et al., 2008; TOMASINI; ALVES, 2007).

Frade et al. (2015) ao discorrer sobre o idoso institucionalizado afirma que os problemas de saúde que a depressão acarreta, bem como, os motivos ou dificuldade da família de assegurar estes idosos devem ser compreendidos para que possa ser oferecido o melhor cuidado e condições possíveis, que objetivam diminuir consideravelmente as implicações relacionadas à institucionalização na saúde do idoso. As instituições que recebem os idosos têm de implementar ações que reduzam o peso da institucionalização, através de atividades diversificadas que exercitem a capacidade física e mental do idoso, por exemplo.

A institucionalização do idoso é uma opção muito procurada quando os familiares não aceitam a função de cuidadores, devido a instabilidade econômica e dependência física do idoso. $\mathrm{O}$ idoso ao ser institucionalizado é obrigado a adaptar-se as rotinas de horários, a divisão do ambiente com pessoas desconhecidas, distância da família dentre outros, o que pode acarretar em problemas psicológicos (PORCU et al., 2002).

A institucionalização de idosos é um assunto delicado, pois a aceitação muitas vezes não é consensual. Para um suporte adequado ao idoso, é imprescindível traçar o perfil do idoso, distinguindo os que vivem um envelhecimento bem-sucedido daqueles que necessitam de atenção profissional especializada (PESTANA; SANTO, 2008).

O idoso ao ser institucionalizado passa por processos de vulnerabilidades social, econômica e psicológica. Nestas instituições podemos encontrar idosos moradores de rua, em condições financeiras precárias, a ausência de aposentadoria por idade ou de benefício de prestação continuada. Alguns idosos não tem casa própria ou possibilitados de alugar e há o abandono familiar. Outras situações encontradas são as diversidades de problemas de saúde e de condições socioeconômicas tanto do idoso quanto da instituição que o abriga, o que às vezes acentua seu grau de vulnerabi- 
lidade física, psíquica ou social devido à falta de preparo dos profissionais que ali atuam (GOLDIN, 2002). Estima-se que $15 \%$ dos idosos manifestam alguns sinais de depressão, correspondendo a $2 \%$ do tipo grave. Em algumas populações, a presença é mais ampla, alcançando de $5 \%$ a $13 \%$ dos pacientes hospitalizados e de $12 \%$ a $16 \%$ nas instituições de longa permanência (SIQUEIRA et al., 2009).

\section{Depressão}

A depressão é uma alteração afetiva, sendo a mais estudada e comentada na atualidade, podendo ser classificada como um transtorno de humor, onde modifica a percepção das pessoas a respeito de si mesmas, enxergando seus problemas como grandes catástrofes. Entre os principais sintomas encontram-se a apatia, irritabilidade, perda de interesse, tristeza, desolação, ideias agressivas dentre outros (ESTEVES; GALVAN, 2006).

A depressão consiste em um dos transtornos psiquiátricos mais comuns entre pessoas idosas, sendo mais frequente entre idosos doentes ou institucionalizados. A depressão nesses indivíduos produz uma influência negativa na sua vida como um todo, sendo que quanto maior for a gravidade do quadro, somando-se a falta de tratamento adequado, pior será o prognóstico. Podemos ressaltar ainda que os idosos com depressão tendem a ter maior comprometimento físico, social e funcional, impactando a sua qualidade de vida (BRASIL, 2007).
Os sintomas de depressão em idosos institucionalizados são frequentes, ocorrendo mais em mulheres do que em homens, indivíduos com mais idade, que apresentem algum tipo de dependência ou alguma insatisfação com a instituição, sendo que este tipo de observação auxilia o enfermeiro na proposta de ações de prevenção, na identificação precoce da depressão e no tratamento (SILVA et al., 2012).

Nos anos 1990, a depressão foi a quarta causa específica de incapacitação, sendo que a projeção para 2020, é que será a segunda causa nos países desenvolvidos e a primeira nos países em desenvolvimento. A depressão é mais frequente nos anos que antecedem a aposentadoria, diminuindo na década seguinte e aumentando após os 75 anos (BRASIL, 2007).

No que tange aos fatores de riscos da depressão, destaca-se que a dificuldade nas relações pessoais, o isolamento, os conflitos com a família ou com outras pessoas, as dificuldades econômicas e outros fatores de estresse. Outros fatores podem agravar o quadro de depressão nos idosos, como por exemplo, o uso de álcool, sintomas psicóticos como alucinações, transtorno de personalidade, ausência de apoio social, a não aceitação do diagnóstico, a não aderência às recomendações terapêuticas, dentre outros (BRASIL, 2007). 


\section{Papel do profissional de enfermagem}

O enfermeiro deve estar preparado para trabalhar com uma demanda diversificada, especialmente quando ligado as questões psicológicas dos idosos, que se desconhecidas proporcionam dificuldades para prevenir, diagnosticar e realizar $o$ tratamento adequado. $\mathrm{O}$ enfermeiro necessita obter o conhecimento sobre os sintomas da depressão no intuito de acolher integralmente e direcionar para a prevenção do transtorno (TESTON; CARREIRA; MARCON, 2014).

As instituições que recebem os idosos necessitam de uma equipe de profissionais que desenvolvam um trabalho focado no cuidado adequado aos residentes, proporcionando qualidade de vida. $\mathrm{O}$ enfermeiro tem um papel de tornar o cuidado mais humanizado, acolhedor e integral (SANTOS et al., 2008).

Para garantir a melhor preparação de um enfermeiro, Lopes (2007) coloca a importância do SAE (Sistema da Assistência da Enfermagem), que possibilita aprimoramento e desenvolvimento do cuidado. Esse sistema é constituído por uma dinâmica de 5 etapas correlacionadas: investigação, diagnóstico, planejamento, implementação e avaliação onde o não seguimento destas etapas pode comprometer o trabalho.

\section{Materiais e métodos}

Foi realizada uma revisão bibliográfica sobre o índice de depressão em idosos institucionalizados. Os métodos para elaboração de revisões sistemáticas preveem: elaboração da pergunta de pesquisa; busca na literatura; seleção dos artigos; extração dos dados; avaliação da qualidade metodológica; síntese dos dados; avaliação da qualidade das evidências; redação e publicação dos resultados (SAMPAIO; MANCINI, 2007).

Para levantamento do material da pesquisa foram utilizados os seguintes bancos de dados: SCIELO (Scientific Electronic Library Online), MEDLINE (Literatura Internacional em Ciências da Saúde), indexados no site da Biblioteca Virtual de Saúde (B.V.S), e utilizados oito artigos científicos completos publicados entre 2009 e 2017, que abordavam a temática e que atingissem os objetivos da pesquisa. Os artigos foram levantados utilizando os Descritores em Ciência da Saúde (DECs): depressão, idosos, institucionalização e enfermagem e, em inglês Depression, Elderly, Institutionalization e Nursing. Os critérios de exclusão foram artigos publicados antes ou após as datas selecionadas, resumos, artigos incompletos, teses e dissertações. Por meio da leitura dos títulos e resumos de artigos, foram selecionados os que tinham maior concordância com o tema em questão.

\section{Resultados e discussão}

O Quadro 1 traz informações específicas de cada estudo selecionado, apresentando suas principais conclusões. 
Quadro 1. Informações detalhadas de cada estudo referente ao título do artigo, autores, ano e principais conclusões.

\begin{tabular}{|c|c|c|c|}
\hline Título do artigo & Autor & Ano & $\begin{array}{l}\text { Principais } \\
\text { conclusões }\end{array}$ \\
\hline $\begin{array}{l}\text { Depressão e perfis sócios demo- } \\
\text { gráficos e clínicos de idosos institu- } \\
\text { cionalizados sem déficit cognitivo }\end{array}$ & $\begin{array}{l}\text { GALHARDO; } \\
\text { MARIOSA; TAKATA }\end{array}$ & 2009 & $\begin{array}{l}\text { Este estudo mostrou que o } \\
\text { principal motivo da instituciona- } \\
\text { lização é a ausência de suporte } \\
\text { familiar ( } 74 \%) \text {. }\end{array}$ \\
\hline $\begin{array}{l}\text { Análise da sintomatologia depressi- } \\
\text { va nos moradores do Abrigo Cristo } \\
\text { Redentor através da aplicação da } \\
\text { escala de depressão geriátrica } \\
\text { (EDG) }\end{array}$ & SIQUEIRA et al. & 2009 & $\begin{array}{l}\text { O estudo apresentou que nos } \\
\text { idosos avaliados pelo autor no } \\
\text { abrigo Cristo Redentor foram } \\
\text { identificados } 51 \% \text { de casos de } \\
\text { depressão entre os } 55 \text { idosos } \\
\text { avaliados. }\end{array}$ \\
\hline $\begin{array}{l}\text { Prevalência de depressão em ido- } \\
\text { sos institucionalizados }\end{array}$ & SIQUEIRA et al. & 2011 & $\begin{array}{l}61,60 \% \text { dos idosos instituciona- } \\
\text { lizados apresentaram sintomas } \\
\text { depressivos. Dentre estes, } \\
36,60 \% \text { estavam instituciona- } \\
\text { lizados há menos de um ano. } \\
\text { Isso pode ocorrer em virtude } \\
\text { da saída do lar e/ou da família } \\
\text { para uma instituição asilar, oca- } \\
\text { sionando grandes mudanças e } \\
\text { baixa expectativa em sua vida. }\end{array}$ \\
\hline $\begin{array}{l}\text { Depressão em idosos institucionali- } \\
\text { zados no distrito de Bragança }\end{array}$ & VAZ; GASPAR & 2011 & $\begin{array}{l}\text { O estudo mostrou que a taxa } \\
\text { de prevalência de depressão } \\
\text { foi de } 46,7 \% \text {, da qual } 33,3 \% \\
\text { apresentaram depressão ligei- } \\
\text { ra e } 13,4 \% \text { depressão grave. } \\
\text { Em relação à prevalência da } \\
\text { depressão segundo o sexo, foi } \\
\text { constatado maior índice nas } \\
\text { mulheres do que nos homens } \\
\text { (50,9\% vs } 40,3 \%) \text {. O nível de } \\
\text { depressão apresentou maior } \\
\text { elevação em idosos com maior } \\
\text { solidão. O nível de depressão } \\
\text { tende a ser mais elevado em } \\
\text { idosos mais dependentes nas } \\
\text { atividades de vida diária. }\end{array}$ \\
\hline
\end{tabular}




\begin{tabular}{|c|c|c|c|}
\hline $\begin{array}{l}\text { Elderly institutionalization and de- } \\
\text { pression }\end{array}$ & RUNCAN & 2012 & $\begin{array}{l}\text { O artigo traz que a depressão } \\
\text { no idoso é algo comum, no en- } \\
\text { tanto ganha força quanto este é } \\
\text { institucionalizado, pois, quando } \\
\text { este é forçado a mudar de resi- } \\
\text { dência ou até mesmo por von- } \\
\text { tade própria tende a sofrer uma } \\
\text { pressão ambiental, o que por } \\
\text { sua vez desencadeia perda de } \\
\text { interesse em atividades cotidia- } \\
\text { nas e isolamento social. }\end{array}$ \\
\hline $\begin{array}{l}\text { Depressão no idoso: sintomas em } \\
\text { indivíduos institucionalizados e } \\
\text { não-institucionalizados }\end{array}$ & FRADE et al. & 2015 & $\begin{array}{l}\text { Os sintomas de depressão fo- } \\
\text { ram mais prevalentes no grupo } \\
\text { dos idosos institucionalizados } \\
\text { (média }=18,12 \text { ), em compara- } \\
\text { ção com os não instituciona- } \\
\text { lizados (média = 11,46), com } \\
\text { diferenças estatisticamente } \\
\text { expressivas. Os sintomas de } \\
\text { depressão foram prevalentes } \\
\text { nos idosos que vivem sozinhos } \\
\text { (viúvos e solteiros), quando } \\
\text { comparados com o grupo dos } \\
\text { idosos que vivem com o côn- } \\
\text { juge. Os idosos solteiros e/ou } \\
\text { viúvos, independentemente } \\
\text { de estar institucionalizados ou } \\
\text { não, aparentam maior tendên- } \\
\text { cia para desenvolver sintomas } \\
\text { depressivos. }\end{array}$ \\
\hline $\begin{array}{l}\text { Instrumentos desenvolvidos para o } \\
\text { gerenciamento e cuidado de idosos } \\
\text { em instituições de longa perma- } \\
\text { nência: uma revisão sistemática }\end{array}$ & MEDEIROS et al. & 2016 & $\begin{array}{l}\text { A depressão, a cognição e a } \\
\text { capacidade funcional, foram, } \\
\text { as variáveis mais identificadas } \\
\text { nos estudos. A alta prevalência } \\
\text { de depressão no contexto ins- } \\
\text { titucionalização está frequen- } \\
\text { temente relacionada a fatores } \\
\text { como limitação e/ou dependên- } \\
\text { cia e insatisfação com a institui- } \\
\text { ção. É importante identificar a } \\
\text { presença de sinais e sintomas } \\
\text { que remetam a problemas de } \\
\text { comportamento, sentimentos } \\
\text { negativos e ausência de bem- } \\
\text {-estar, através de comunicação } \\
\text { verbal e não verbal. }\end{array}$ \\
\hline
\end{tabular}




\begin{tabular}{|c|c|c|c|}
\hline $\begin{array}{l}\text { Depressão em idosos instituciona- } \\
\text { lizados: padrões cognitivos e quali- } \\
\text { dade de vida }\end{array}$ & $\begin{array}{l}\text { HARTMANN JU- } \\
\text { NIOR; GOMES }\end{array}$ & 2016 & $\begin{array}{l}\text { A média de idade dos partici- } \\
\text { pantes foi de } 78 \text { anos. Quanto } \\
\text { ao gênero, houve predomínio } \\
\text { de mulheres } 89,6 \% \text { as quais, } \\
31,3 \% \text { eram solteiras ou viú- } \\
\text { vas. Dentre os homens, } 40,0 \% \\
\text { eram solteiros e viúvos. Em } \\
59,4 \% \text { idosos havia pontuação } \\
\text { compatível com história de } \\
\text { depressão, e que } 36,4 \% \text { idosos } \\
\text { estavam deprimidos na ocasião } \\
\text { da coleta dos dados. Não foi } \\
\text { verificado diferença significante } \\
\text { na associação entre depressão } \\
\text { passada e inicial. A presença } \\
\text { de depressão foi mais frequen- } \\
\text { te em idosos iletrados, que não } \\
\text { recebiam visitas familiares, que } \\
\text { não saíam da instituição, não } \\
\text { se submetiam aos exames de } \\
\text { saúde de rotina e tinham exer- } \\
\text { cido, no passado, ocupações } \\
\text { de menor complexidade. }\end{array}$ \\
\hline $\begin{array}{l}\text { O papel do enfermeiro na recupe- } \\
\text { ração de idosos depressivos }\end{array}$ & TREVISAN et al. & 2016 & $\begin{array}{l}\text { Os principais sintomas da de- } \\
\text { pressão evidenciados foram: a } \\
\text { diminuição da capacidade de } \\
\text { concentração, fadiga acentu- } \\
\text { ada, insônia, falta de apetite, } \\
\text { perda de interesse pelas coisas } \\
\text { que antes eram prazerosas } \\
\text { (anedonia), baixa autoestima, } \\
\text { insegurança, isolamento social, } \\
\text { humor depressivo, sentimento } \\
\text { de culpa e tentativa de suicídio. }\end{array}$ \\
\hline $\begin{array}{l}\text { Fatores associados a depressão } \\
\text { e os cuidados de enfermagem no } \\
\text { idoso }\end{array}$ & VENTURA et al & 2016 & $\begin{array}{l}\text { A depressão é uma das } \\
\text { afecções mais comuns nos } \\
\text { indivíduos idosos com grande } \\
\text { influência na qualidade de vida } \\
\text { destes, os avanços tecnológi- } \\
\text { cos e consequente aumento na } \\
\text { expectativa de vida trazem con- } \\
\text { sigo a insatisfação de aspectos } \\
\text { psicológicos nesta população. }\end{array}$ \\
\hline
\end{tabular}

Embora tenha-se conseguido grandes avanços das ciências da saúde, nos últimos tempos e como consequência o aumento da longevidade, a realidade mostra que os últimos anos de vida são, muitas vezes, acompanhados de situações de fragilidade e de incapacidade (VENTURA, et al., 2016). 
Runcan (2012) traz em seu estudo que a depressão no individuo senil é algo latente e se potencializa quando este é residente de instituições, pois tendem a sentirem se tristes, pessimistas, experimentam sentimentos de fracasso, estão insatisfeitos o que leva a restrição de contatos sociais, perda de interesse em várias atividades, com foco para a morte e pensamentos suicidas.

Aliado ao descrito acima Frade et $a l .$, (2015) descrevem que a maior prevalência de depressão em idosos institucionalizados está ligada a fatores como ausência de suporte familiar, limitação e/ou dependência, insatisfação com a instituição e a solidão, como em idosos que apresentam se viúvos ou solteiros. Dentre os idosos institucionalizados com depressão, a maioria são do gênero feminino, predominando também em idosos solteiros ou viúvos, de acordo com o Quadro 1. A frequência da depressão foi enfatizada em idosos que não recebiam visitas familiares, que não saíam da instituição, não faziam exames de rotina e que haviam exercido no passado ocupações de menor complexidade.

Obteve-se uma elevada taxa de prevalência de depressão, a qual afeta cerca da metade dos idosos institucionalizados (HARTMANN JUNIOR; GOMES, 2011; SIQUEIRA et al., 2009; SIQUEIRA et $a l ., 2011)$.

). Também foi considerado que o nível de depressão tende a ser mais elevado em idosos que apresentam se mais dependentes nas atividades de vida diária. Como já citado anteriormente as mudanças ambientais são os principais desencadeantes da depressão, assim como a baixa autoestima, isolamento social, insegurança, dentre outros, e Siqueira, et al (2011) em seu estudo trazem que $36,6 \%$ dos idosos institucionalizados a menos de 1 ano apresentam sintomas depressivos.

Oliveira, Santos e Pavarini (2014), pesquisando sobre sintomas depressivos em idosos residentes nas ILPIS de São Carolos, São Paulo, realizou entrevista individuais com 107 idosos entre 60 a 80 anos ou mais. Dos idosos na faixa etária de 60 a 69 anos, encontraram sintomas depressivos em 58\%, nos de 70 a 79 anos, houve sintomas depressivos em $52 \%$ e, nos com idade igual ou superior a 80 anos foi encontrado $83 \%$ com sintomas depressivos, destaca-se ainda que existe uma predominância de idosos do sexo feminino $(61 \%)$, viúvos (48\%) e a média e idade desses idosos foram de 77 anos.

Para reduzir o índice de depressão dos idosos institucionalizados, o papel do profissional de enfermagem é de extrema importância. Silva et al. (2009) (2015), pesquisando sobre os sintomas depressivos, relata que a pessoa idosa pode apresentar sintomas de depressão mascarados os quais podem ser confundidos, pelos profissionais de saúde que o atendem, como características próprias do processo fisiológico de envelhecimento, dificultando a obtenção de um diagnóstico preciso e rápido. Dessa maneira, faz-se necessário à implementação de uma dialogicidade na presença ou ausência da depressão, para isso há necessidade de capacitação dos enfermeiros para 
realizarem, de forma adequada, esta identificação de sintomas da depressão.

A contribuição do enfermeiro é de significativa importância para a recuperação do paciente depressivo, atuando na orientação sobre a importância da terapia medicamentosa, esclarecimento de dúvidas, estimulando o crescimento pessoal e o desempenho de novos papéis na sociedade, incentivando a prática de exercícios físicos e a participação em grupos da terceira idade (TREVISAN et al., 2016).

\section{Considerações finais}

Os idosos institucionalizados apresentaram uma maior prevalência de depressão, ligados a fatores como ausência de suporte familiar, limitação e/ ou dependência, insatisfação com a instituição, e também o nível de depressão foi maior em idosos com mais solitários. Pode-se afirmar que, dentre os idosos institucionalizados com depressão, a maioria são do gênero feminino, solteiros ou viúvos, ou que não recebiam visitas familiares, que não saíam da instituição, não faziam exames de rotina, que tinham exercido no passado ocupações de menor complexidade.

Destaca-se, que para diminuir o índice de depressão dos idosos institucionalizados, o papel do profissional de enfermagem é de extrema importância, visto que o enfermeiro é o profissional que trata do conjunto de técnicas e procedimentos voltados para o bem-estar do doente, logo, faz-se necessário que este tenha habilidades e conhecimento para reconhecer e intervir apropriadamente nos casos em que o indivíduo está em sofrimento psíquico.

Depression in institutionalized elderly people: systematic review

\section{Abstract}

The institutionalization of the elderly is an option quite popular when family members do not accept the caregivers role. The elderly, when institutionalized, are forced to adjust their schedules, share the environment with unknown people, distance from the family, and others, and can be the starting point for psychological problems, such as depression. The objective of this study was to identify if the institutionalized elderly present depression. To carry out the study, a systematized bibliographical research was carried out, covering scientific articles, complete and/or syntheses, publications were selected between the years 2009 to 2017. According to the results found, in the majority of the studies analyzed, presented a higher prevalence of depression in institutionalized elderly people, related to factors such as absence of family support, limitation and / or dependence, dissatisfaction with the institution and loneliness, the main symptoms of depression evidenced were lack of appetite, marked fatigue, low self-esteem, insecurity, decreased ability to concentrate, social isolation, among others. It can be stated that, among the institutionalized elderly with depression, the majority of the elderly are of the female gender, being also emphasized in elderly people who did not receive family visits, who did not leave the institution, did not perform routine exams that had exercised in the past occupations of less complexity. Thus, it is necessary that the professionals involved in care for the institutionalized elderly have the skills and 
knowledge to recognize and intervene appropriately in cases where the individual is in psychological distress.

Keywords: Depression. Elderly. Institutionalization. Nursing.

\section{Referências}

BELTRÃO, K. I.; CAMARANO, A. A.; KANSO, S. Dinâmica populacional brasileira na virada do século $X X$. Rio de Janeiro: IPEA, 2004.

BRASIL. Agência Nacional de Vigilância Sanitária. Regulamento técnico que define as normas de funcionamento para as instituições de longa permanência para idosos. RDC nº 283. 2005. Disponível em: <http://portal. anvisa.gov.br/documents/10181/2718376/ RDC_283_2005_COMP.pdf/a38f2055-c23a-4eca-94ed-76fa43acb1df>. Acesso em: 6 nov. 2017.

BRASIL. Instituto Brasileiro de Geografia e Estatística - IBGE. PNAD 2016: população idosa cresce $16,0 \%$ frente a 2012 e chega a 29,6 milhões. 2017. Disponível em: <https:// agenciadenoticias.ibge.gov.br/agencia-sala-de-imprensa/2013-agencia-de-noticias/ releases/18263-pnad-2016-populacao-idosa-cresce-16-0-frente-a-2012-e-chega-a-29-6-milhoes>. Acesso em: 20 jan. 2019.

BRASIL. Ministério da Saúde; Secretaria de Atenção à Saúde. Envelhecimento e saúde da pessoa idosa [Internet]. Brasília; 2007. Disponível em: <http://bvsms.saude.gov.br/ bvs/publicacoes/abcad19.pdf $>$. Acesso em: 19 abr. 2017.

BRASIL. Organização Pan-Americana da Saúde. Envelhecimento ativo: uma política de saúde. Brasília-DF, 2005.

CARREIRA, L. et al. Prevalência de depressão em idosos institucionalizados. Revista de Enfermagem, Rio de Janeiro, v.19, n. 2, p. 268-273, jun. 2011.
CREUTZBERG, M. et al. Instituição de longa permanência para idosos: a imagem que permanece. Texto \& Contexto de Enfermagem, Florianopolis, v. 17, n. 2, p. 273-279, jun. 2008.

DIAS, A. M. O processo de envelhecimento humano e a saúde do idoso nas práticas curriculares do curso de fisioterapia da UNIVALI campus Itajaí: um estudo de caso. 2007. 189 f. Dissertação (Mestrado em Saúde e Gestão do Trabalho) - Universidade do Vale do Itajaí, 2007.

ESTEVES, F. C.; GALVAN, A. L. Depressão numa contextualização contemporânea. Aletheia, n. 24, p. 127-135, jul./dez. 2006.

FRADE, J. et al. Depressão no idoso: sintomas em indivíduos institucionalizados e não-institucionalizados. Revista de Enfermagem Referencia, série IV, n. 4, p. 41-49, jan./fev. 2015.

FRIAS, S. R. Instituições de longa permanência para idosos. 2015. Disponível em: <http:// www.unatiuerj.com.br/Cartilha\%20ILPI\%20 FINAL\%20PDF.pdf>. Acesso em: 6 nov. 2017.

GALHARDO, V. A. C.; MARIOSA, M. A. S.; TAKATA, J. P. I. Depressão e perfis sociodemográfico e clínico de idosos institucionalizados sem déficit cognitivo. Revista de Medicina, Belo Horizonte, v. 20, n. 1, p. 16-21, 2009.

GIL, A. C. Métodos e técnicas de pesquisa social. 6. ed. São Paulo: Atlas, 2008.

GOLDIM, J. R. Bioética e envelhecimento. In: FREITAS, E. V.; PY, L.; NÉRI, A. L.; CANCADO, F. A. X.; GORZONI, M. L.; ROCHA, S. M. Tratado de geriatria e gerontologia. Rio de Janeiro: Guanabara Koogan; 2002. p. 85-99.

HARTMANN JUNIOR, J. A. S.; GOMES, G. C. Depressão em idosos institucionalizados: padrões cognitivos e qualidade de vida. Ciências \& Cognição, Rio de Janeiro, v. 21, n. 1, p. 137-154, 2016.

HARTMANN JUNIOR, J. A. S.; GOMES, G. C. Depressão em idosos institucionalizados 
as singularidades de um sofrimento visto em sua diversidade. Revista SBPH[online], Rio de Janeiro, v. 17, n. 2, p. 83-105, dez. 2014.

JACOB FILHO, W. Promoção da saúde do idoso. São Paulo: Lemos Editorial, 1998.

LOPES, F. L. et al. Diagnósticos de enfermagem de idosos residentes em uma instituição de longa permanência (ILP). Ciência e Cuidado de Saúde, Maringá, v. 6, n. 1, p. 59-67, jan./mar. 2007.

MARTINS, R. A. Depressão no idoso. Revista Millenium, v. 13, n. 34, p. 119-123, abr. 2008.

MEDEIROS, P. A. et al. Instrumentos desenvolvidos para o gerenciamento e cuidado de idosos em instituições de longa permanência: uma revisão sistemática Revista Ciência \& Saúde Coletiva, Rio de Janeiro, v. 21, n. 11, p. 3597-3610, 2016.

NETO, M. J.; CORTE-REAL, J. A pessoa idosa institucionalizada: depressão e suporte social. 2011. Disponível em: <http://www. spgg.com.pt/UserFiles/file/A\%20pessoa\%20 idosa\%20institucionalizada.pdf $>$. Acesso em: 3 abr. 2017.

OLIVEIRA, S. C.; SANTOS, A. A.; PAVARINI, S. C. Relação entre sintomas depressivos e a funcionalidade familiar de idosos institucionalizados. Revista de Escola de Enfermagem da USP, São Paulo, v. 48, n. 1, p. 65-71, fev. 2014.

PESTANA, L. C.; SANTO, F. H. E. As engrenagens da saúde na terceira idade: um estudo com idosos asilados. Revista de Escola de Enfermagem da USP, São Paulo, v. 42, n. 2, p. 268-275, jun. 2008.

PORCU, M. et al. Estudo comparativo sobre a prevalência de sintomas depressivos em idosos hospitalizados, institucionalizados e residentes na comunidade. Revista Acta Scientiarum, Maringá, v. 24, n. 3, p. 713717, 2002.

RUNCAN, P. L. Elderly institutionalization and depression. Procedia-social and Behavioral Sciences, v. 33, p. 109-113, 2012.
SAMPAIO, R. F.; MANCINI, M. C. Estudos de revisão sistemática: um guia para síntese criteriosa da evidência científica. Revista Brasileira de Fisioterapia, São Carlos, v.11, n.1, p.83 - 89, jan./fev. 2007.

SANTOS, S. S. C. et al. O papel do enfermeiro na instituição de longa permanência para idosos. Revista de Enfermagem UFPE on line, Recife, v.02, n. 3, p. 291-299, jul./ set. 2008.

SILVA, E. R. et al. Prevalência e fatores associados à depressão entre idosos institucionalizados: subsídio ao cuidado de enfermagem. Revista de Escola de Enfermagem da USP, São Paulo, v. 46, n. 6, p. 1387-1393, dez. 2012.

SILVA, D. S. et al. Reconhecimento e intervenção de enfermagem na depressão do idoso institucionalizado. XIII Encontro Latino Americano de Iniciação Científica e IX Encontro Latino Americano de Pós-Graduação - Universidade do Vale do Paraíba, 2009.

SILVA, J. R.; SILVA, H. G.; RABELO, S. N.; ALEXANDRE, S. G.; FREITAS, M. C. Cuidado de enfermagem ao idoso institucionalizado e deprimido. IV CONGRESSO INTERNACIONAL DE ENVELHECIMENTO HUMANO... Anais CIEH, Campina Grande, v. 2, n. 1, 2015. Disponível em: <http://www.editorarealize.com.br/artigo/visualizar/12252>. Acesso em: 3 abr. 2017.

SIQUEIRA, G. R. et al. Análise da sintomatologia depressiva nos moradores do Abrigo Cristo Redentor através da aplicação da escala de depressão geriátrica (EDG). Ciência \& Saúde Coletiva [online], Rio de Janeiro, v. 14, n. 1, p. 253-259, jan./fev. 2009.

STELLA, F. et al. Depressão no idoso: diagnóstico, tratamento e benefícios da atividade física. Motriz, Rio Claro, v. 8, n. 3, p. 91-98, ago./dez. 2002.

TESTON, E. F.; CARREIRA, L.; MARCON, S. S. Sintomas depressivos em idosos: comparação entre residentes em condomínio especifico para idoso e na comunidade. Revista 
Brasileira de Enfermagem, Brasília, v. 67, n. 3, p. 450-460, 2014.

TRAVASSOS, J. A. Responsabilidade corporativa: institucionalização e ideologia. 2012. 93 f. Dissertação (Mestrado em Ciências Sociais) - Universidade Federal de Minas Gerais, Belo Horizonte, 2012.

TREVISAN, M. et al. O papel do enfermeiro na recuperação de idosos depressivos. Revista Eletrônica Gestão \& Saúde, Brasília, v. 7, n. 1, p. 428-440, out. 2015.

TOMASINI, S. L. V.; ALVES, S. Envelhecimento bem-sucedido e o ambiente das instituições de longa permanência. Revista Brasileira de Ciências do Envelhecimento Humano, Passo Fundo, v. 4, n. 1, p. 88-102, jan./jun. 2007.

VAZ, S. F. A.; GASPAR, N. M. S. Depressão em idosos institucionalizados no distrito de Bragança. Revista de Enfermagem Referência, v. 3, n. 4, p. 49-58, jul. 2011.

VENTURA, J. et al. Fatores associados a depressão e os cuidados de enfermagem no idoso. Revista de Enfermagem, Westphalen, v. 12 , n. 12 , p. 100-113, 2016. 